This is a preprint - please ask before citing

\title{
Psychiatric Progress and The Assumption of Diagnostic Discrimination
}

\author{
Kathryn Tabb
}

\begin{abstract}
The failure of psychiatry to validate its diagnostic constructs is often attributed to the prioritizing of reliability over validity in the structure and content of the Diagnostic and Statistical Manual of Mental Disorders (DSM). Here I argue that in fact what has retarded biomedical approaches to psychopathology is unwarranted optimism about diagnostic discrimination: the assumption that our diagnostic tests group patients together in ways that allow for relevant facts about mental disorder to be discovered. I consider the Research Domain Criteria (RDoC) framework as a new paradigm for classifying objects of psychiatric research that solves some of the challenges brought on by this assumption.
\end{abstract}

\section{Introduction}

It is often said that the architects of the third edition of the Diagnostic and Statistical Manual of Mental Disorders (1980), a task force of the American Psychiatric Association (APA), sacrificed validity for reliability when constructing the manual's categories (Vaillant 1984; Andreasen 2007). According to this view, the DSM went wrong when it adopted an operationalist stance focusing on atheoretical observational criteria, an ecumenical approach that made applying diagnoses simple but learning about them difficult. Without an understanding of underlying mechanisms, some believe, psychiatry has not been able to identify disease entities akin to those in the rest of medicine (Murphy 2006). This narrative suggests that psychiatrists were forced to adopt the $D S M$ 's operationalism, and became uninterested in or unable to pursue etiological explanations. 
this is a preprint - please ask before citing

A better way to put it is that psychiatrists have followed the $D S M$ in assuming that reliability will ultimately lead to validity — that is, that reliable diagnostic categories will demarcate the best targets for validation. Here I argue that rather than the commitment to operationalism, what has been most detrimental to research has been psychiatry's deference to the nosological project itself. The DSM's unfortunate bequest to twentieth- and twenty-first-century psychiatry has been its commitment to the assumption of diagnostic discrimination, which I define as the assumption that our diagnostic tests ${ }^{1}$ group patients together in ways that allow for relevant facts about mental disorder to be discovered. For the purposes of this discussion, relevant facts are those about the underlying mechanisms causing the signs and symptoms that patients present with, of the sort that biomedical researchers hope to find: genetic signatures, neurological or cognitive dysfunctions, focal brain lesions, etc. Facts, in other words, that contribute to the scientific project of "mapping the domain" of mental illness (Poland and Eckardt 2013).

I borrow the term discrimination from psychometrics, where it is defined as the statistical assessment of how a diagnostic test compares with a gold-standard, measured by the test's specificity, sensitivity, predictive value, and likelihood ratios (Knottnerus and Buntinx 2009). In my argument, however, discrimination is invoked as an aspirational term, signifying an ideal rather than a measure: I make the case that while there is often no ground on which to judge diagnostic criteria as discriminative for research purposes, they are often assumed to be so anyway. It is, of course, an empirical question to what extent any $D S M$ criteria facilitate or hinder scientific progress. My aim is not to offer any empirical

\footnotetext{
${ }^{1}$ By "tests" I refer to either the diagnostic criteria of the DSM itself or diagnostic screens based on these criteria. Obviously diagnostic discrimination could be proposed about other diagnostic methods (e.g., the Psychodynamic Diagnostic Manual) but my focus here is on the DSM.
} 
assessments but rather to argue that as long as diagnostic discrimination is broadly assumed, there is reason to think that psychiatric research will, on the whole, be hampered.

The $D S M$ 's central role in research, particularly in guiding the selection of test populations and establishing targets for explanation, is not only entrenched by historical precedent but also held firm by the hand of the biomedical marketplace, in which funding bodies prefer research that directly meets perceived clinical needs. This has led to a focus on the iterative validation of diagnostic constructs, especially the search for etiologies that can inspire new therapies, and the neglect of research that cross-cuts or challenges existing diagnostic boundaries. If the $D S M$ 's categories are discriminative in the relevant sense, such a narrowing of focus is a boon to research. If not, the DSM is analogous to the lamppost in the tale of the man who makes the mistake of looking for his keys where the light is, instead of where he lost them.

In the following section (2) I expand on the idea of diagnostic discrimination, and offer historical and empirical arguments for why we should be pessimistic about whether the $D S M$ 's criteria are discriminative in the relevant way for biomedical research. While (as I suggest in my conclusions) most philosophers have not attended to the inhibiting and restrictive effects of the assumption of diagnostic discrimination, psychiatrists at the National Institute of Mental Health (NIMH) have recently recognized their importance. As I discuss in Section 3, the organization has introduced an alternative classification system for psychiatric research, the Research Domain Criteria (RDoC) project, which aims to validate other sorts of constructs than diagnostic categories. I consider the RDoC here to show one way in which psychiatry could proceed which would justify a greater optimism about the 
discrimination of its measurements. In Section 4 I consider some possible responses to my argument.

\section{The assumption of diagnostic discrimination in psychiatry}

In this section I explore whether the origins, structure, or performance of the DSM's categories give reason to think that they categorize patients into groups about which facts of the sort that interest biomedical researchers can be gathered. The aim of the first edition of the DSM (1952) was to collect statistical information, originally for military purposes. Ever since ambitious task forces have attempted to revise the DSM on to serve contemporary goals, rather than the needs of decades' old census projects. The architects of the DSM-III prioritized the construction of diagnostic categories based on "distilled clinical research experience" as the "first and crucial taxonomic step" (Feighner et al. 1972, 57) towards identifying valid constructs. However Feighner et al.'s constructs were in fact an amalgam of data and received clinical intuition, and many of the basic taxonomic divisions were inherited unchallenged (Kendler 2009). Similarly, the architects for the DSM-5 announced the need to "transcend the limitations of the current DSM paradigm" (Kupfer, First, and Regier 2008 p. xxii). In the end, however, with some exceptions, the nosological structure remained relatively stable.

Turning to the manual's structure, it is clear that if etiopathogenic facts about mental disorders are forthcoming, they will not stand in simple causal relationships to the signs and symptoms that act as diagnostic criteria. As of its third edition the DSM's categories have been polythetic, requiring patients to present with only $n$ symptoms out of a longer list in order to meet the threshold for the disorder. Pragmatic factors also increase 
heterogeneity; for example, screens for psychopathology tend to have low thresholds, since the cost of a false-negative (abandoning a patient in need of care) is viewed as higher than a false-positive (giving unneeded treatment) (Ross 2014). As a result, the DSM's criteria allow for incredible diversity — for example, the DSM-5 post-traumatic stress disorder can be diagnosed on the basis of 636,120 different combinations of symptoms (Olbert 2014). This may mean that in fact conspecific patients have different underlying pathologies that cause these similar but distinct manifestations. Biomedical facts about patients sharing diagnoses will explain this diversity either by revealing the homogeneity beneath the promiscuity or by supplying disjunctive accounts of the mechanisms that underlies it. Such symptomatic heterogeneity is frequently found among diagnostic conspecifics in other fields of medicine, such as cancer or lupus, and is not on its own evidence against discrimination - though it can certainly confound research (Millon and Klerman 1986; Poland and Eckardt 2013). But the lack of compelling confirmations of psychiatry's taxonomic boundaries by genetics, epidimiology, neurophysiology, and other allied sciences is worrying, and raises the question of whether the manual is useful for anything more than identifying phenotypic clusters. Taxometric and epidemiological studies reveal that the enormous heterogeneity in symptoms and course actually contain recognizable sub-types that appear more frequently than others, but underlying differences in causal pathways or mechanisms that could explain these trends have not been found (Nandi, Beard, and Galea 2009).

Indeed, the biologization of psychiatric research has not led to the discovery of any laboratory markers for specific psychiatric conditions, and there remain no biological screens for psychopathology; only the checklists of the DSM and other manuals, and the 
tests based on their operationalizations. Decades of research into psychiatric and behavioral genetics has failed to turn up genes specific to particular disorders (though the heritability of types of psychopathology have been demonstrated (Merikangas and Risch 2003)) or neurological mechanisms (despite advances in our understanding of the neurological underpinnings of signs and symptoms (Gillihan and Parens 2011)). The pharmaceutical industry has capitalized on optimism about a one-to-one correspondence between diagnosis, underlying mechanism, and treatment — notable is frequent relabeling of treatments specific to symptoms (e.g., "tranquilizers") as treatments for purported disease entities (e.g., "antipsychotics") — but still nearly all psychopharmaceutical interventions remain nonspecific.

All in all, the current state of the art in biomedical psychiatry cannot justify the assumption of diagnostic discrimination. In the next section I review the claim, held by a growing number of psychiatrists, that the disappointing failure to validate the DSM's constructs is due to the fact that there is nothing to validate. Put in its strongest form, the argument is that the psychiatric kinds being diagnosed by the manual are fictions, or, from the empiricist perspective, useless constructs. Or, in my metaphysically neutral terms, the diagnostic tests for these constructs are not discriminative in the relevant sense, in so far as little of interest from the perspective of biomedicine can be said about diagnostic conspecifics.

\section{An epistemic bottleneck}

A valid taxonomy has long been viewed as the first step in psychiatric research. As described in the previous section, influential theorists of psychiatric validity have imagined 
this is a preprint - please ask before citing

a boot-strapping model, in which the first phase of achieving validity is settling on a clinical description of diagnostic kinds. Andreasen, for example, writes that only "once a reliable method is applied to define symptoms or delineate a potential diagnostic category or dimension of psychopathology" can "these variables then be validated by examining their relationship to external measures" (Andreasen 1995). The DSMs have, historically, provided the independent variable for studies attempting to validate psychiatric kinds.

Recently, however, a new round of critics have suggested that the heterogeneity of test populations collected on the grounds of their DSM diagnoses confounds progress, and some believe the best response is to do away entirely with diagnostic constructs as targets for validation (Hyman and Fenton 2003; Merikangas and Risch 2003). Their thought is that explanations that facilitate intervention and recovery are better found at other levels - for example, the level of the symptom, the gene, or the neural mechanism. Sanislow et al. have written that "dependence on conventional nosologies leaves the enterprise of understanding mechanisms of psychopathology in the awkward position of assuming the validity of single disorders and organizing research accordingly" (2012).

Steven Hyman describes how, as the director of the NIMH in the late 1990s, he became increasingly frustrated with the lack of research into treatments for the devastating cognitive deficits of schizophrenia. He realized taht the lack of interest in cognitive symptoms was due to the bottleneck put on research by the DSM's diagnostic criteria, which do not include cognitive deficits. "Given the status of the DSM-IV criteria as the community consensus," Hyman writes, "the U.S. Food and Drug Administration (FDA) held that it could not, by itself, recognize the cognitive symptoms of schizophrenia as an indication for the development and approval of new treatments" (Hyman 2010, 157). 
this is a preprint - please ask before citing

Recently, the DSM-5 Task Force has justified the continued lack of inclusion of cognitive symptoms quite explicitly, on the grounds that "cognition may not be useful as differential diagnosis tool." 2

Hyman's worry was that a vicious cycle is produced in which the exclusion of a symptom because it is not clinically useful leads to the suppression of precisely the kind of research that would make its saliency for psychiatric practice clear. More broadly, the requirement that signs or symptoms be studied within diagnostic constructs meant that it was hard to gather test populations that targeted objects of investigation below the level of the diagnosis. In 2009, with his colleagues at the NIMH, Hyman began to construct a classification system for research, which would allow scientists to apply for funding from the Institute without structuring their studies around DSM categories. At stake with this new approach was the longstanding assumption that psychiatry's scientific targets should be limited to the same set of diagnostic categories used in clinical practice.

Under RDoC, instead of the DSM's constructs psychiatric investigators present their experiments as targeting fundamental components of mental functioning, or "research domains," that are drawn from allied sciences. These contribute one axis to the matrix that the NIMH has proposed to organize psychiatric research, which is sub-divided into more specific "constructs" — for example, "reward valuation," "performance monitoring," or "attachment formation and maintenance." The other axis is "units of analysis," ranging from "genes" to "behavior." By encouraging the funding of research that investigates a certain research domain(s) at a certain unit(s) of analysis, the $\mathrm{RDoC}$ changes the targets of validation from "clinical endpoints that have remained unchanged for decades" (Hyman

${ }^{2}$ http://www.dsm5.org/ProposedRevision/Pages/proposedrevision.aspx?rid=411\#. 
this is a preprint - please ask before citing

and Fenton 2003, 351) to any sort of phenomenon relevant to psychopathology, either an extreme on a spectrum of human variation or a dysfunctional structure or process.

Rather than seeking to replace the $D S M$ as a diagnostic manual, $\mathrm{RDoC}$ is a classification protocol for researchers that aims to encourage a profound shift in the way research samples are conceived of and assembled. Instead of relying on DSM categories to gather research populations, NIMH researchers gather whatever populations are pertinent to their domain of interest. This method makes unnecessary the roundabouts researchers have always used to precisify generic diagnostic screens to meet their own needs (Meehl 1986; Kutschenko 2011a). One of the aims of RDoC is to allow for the inclusion of patients typically ignored in research because they fall into an "not otherwise specified" category, or are below the threshold for diagnosis. So, for example, a group researching fear circuitry (construct of interest: fear/acute threat, domain: negative valence systems, unit: circuits) might use as their test population patients seeking medical help for anxiety, regardless of whether they meet any specific diagnostic criteria. ${ }^{3}$

The RDoC project avoids the pitfall of assuming diagnostic discrimination, although, as I discuss in Section 4.3, it still relies on other types of discrimination that may be problematic. Of interest here is that in order to move psychiatric research beyond a limited set of targets, the NIMH has placed its bets for discriminative tests outside of the pages of the DSM, putting its faith in researchers to design experiments that use appropriate screens to investigate constructs relevant to psychopathology. While debates over which sorts of objects are most worthy of study may continue to be played out

\footnotetext{
${ }^{3}$ This example is borrowed from the NIMH's online materials about the RDOC - see http://www.nimh.nih.gov/research-priorities/rdoc/nimh-research-domain-criteriardoc.shtml\#toc_studies for the full example. Accessed 6/18/14.
} 
this is a preprint - please ask before citing

through the distribution of funding dollars, these judgments will be constrained by current epistemological and methodological commitments rather than nosological tradition.

\section{Some possible objections}

I have argued that the influence of the DSM over psychiatric research should indeed be blamed, at least in part, for the disappointing failure of psychiatric research to produce robust advances comparable to the other biomedical sciences. ${ }^{4}$ This effect is due the assumption that diagnostic categories are the appropriate grounds on which to draw test populations for research purposes. In this section I consider three possible objections to my argument. The first is that warrant for the belief in diagnostic discrimination can be found in clinical practice. The second is that some assumptions about discrimination are impossible to avoid, and that its bottlenecking effects on progress are a necessary cost of the scientific method. The third is that by giving up on validating the DSM's categories, psychiatry would lose track of its true targets.

\subsection{The Clinical Case for Diagnostic Discrimination}

It has been assumed that if clinicians are able to separate patients into discrete kinds based on their symptomology there is good reason to anticipate that scientific validators will ultimately reinforce these divisions (Robins and Guze 1970). However, studies of the actual usage of the manual suggest that clinicians find it primarily useful for securing treatment options, and mostly ignore its complex polythetic structure (First and Westen 2007). Practitioners engage in diagnostic "bracket creep" to tweak coverage

\footnotetext{
${ }^{4}$ There are, of course, other crucial factors here. The brain is more complex than other medical objects and that explanations of psychopathology from a biomedical perspective may always be
} 
benefits and to duck the restrictions insurance companies put on their ability to utilize their expert judgment on the basis of DSM thresholds (Bowker and Star 1999). In fact, ethnographic research reveals that diagnoses often come after treatment, rather than guiding it (Whooley 2010, 461). If the manual's ubiquity in clinical practice is due to its integral role in the larger machinery of industrial and corporate healthcare rather than its accurate representation of clinical types, any argument for diagnostic discrimination on these grounds is unsound.

Further evidence that the manual's diagnostic constructs do not accurately represent clinical concepts of disorder comes from the widespread alarm over the deprecation of the experience of the patient due to the $D S M$ 's reductive approach to description (Andreasen 2007). The DSM's operationalized descriptions neglect the fact that patients "react to their abnormalities in all kinds of ways that may sometimes require the categories of meaning and experience in order to be understood or explained" (Sass, Parnas, and Zahavi 2011, 16). Some phenomenologically-oriented clinicians and philosophers have suggested that these neglected aspects of mental illness should themselves be targets for validation ((Henriksen and Parnas 2012; Parnas et al. 2005). Taken together, these criticisms suggest that the DSM categories do not reflect the clinical picture sufficiently to justify optimism about their utility in the research setting.

\subsection{The inevitability of diagnostic discrimination}

Another possible objection is that the assumption of discrimination is inevitable in psychiatric investigation, and that the $D S M$ is not (uniquely) culpable. Studies dividing subjects into groups must be always depend on tests assumed to be discriminative for the

(to a greater degree than elsewhere in medicine) incomplete without contributions from 
this is a preprint - please ask before citing

construct in question. Strategies like RDoC, it could be argued, simply replace the diagnostic constructs of the $D S M$ with other sorts of constructs, in this case the subcategories of its proposed domains. The validity of these constructs can surely also be challenged, and the organization of research methods and practices in accordance with them could also be restrictive.

My aim is not to dismiss the importance of discrimination in psychiatric research, nor to suggest that psychiatry can or should do without constructs altogether, but rather to challenge the assumption that the DSM's criteria are discriminative for research purposes. While the RDoC also relies on constructs, its architects have emphasized that these constructs are, first, completely open to revision and, second, explicitly designed to be broad enough to include the leading paradigms within psychiatric research. ${ }^{5}$ Notably, RDoC does not limit the conceivable objects of psychiatric research - such as specific genes, or behaviors - only the loci on the matrix at which the objects fall. Accordingly, researchers have a significant amount of autonomy in the design of their research, and (like in all scientific research) their choice of construct, and the tests they use to measure for it, should be scrutinized closely by their peers.

\subsection{The value of diagnostic kinds for psychiatric research}

A final objection worth considering is whether the gains to research productivity that would come from having discriminative targets have too high an epistemological or

psychology, the social sciences, and even the humanities.

${ }^{5}$ If the NIMH does not fulfill its promise to update the matrix's, it could well end up categories as calcified and restrictive as the $D S M$ 's. 
ethical cost. It has been argued that keeping psychiatry focused on diagnostic kinds is the best way to avoid the reduction of the mentally ill to their component parts, neglecting the phenomenological core of psychopathology in ways dangerous for patients (McLaren 2011; Walter 2013, Poland and Eckardt 2013). There is a risk that the NIMH's own assumptions about the proper targets for psychiatric explanation may become (in Hyman's evocative term for the $D S M)$ another "unintended epistemic prison" $(2010,157)$.

The NIMH has made little secret of its preference for analyses at the level of brain circuits, reasoning that it is at this level that science is most rapidly gaining insight into the underlying correlates of behavior (Insel et al. 2010). This approach has garnered accusations that the RDoC is "mindless" (Frances 2013), that is, aggressively biomedical at the cost of psychological perspectives. In response Bolton (2013) has argued that the NIMH's claim that "all mental diseases are brain diseases" need not be reductionistic insofar as the brain can be seen as integrated into a complex network of causal relations that extend beyond the individual. However, especially in light the NIMH's increasingly enthusiastic pursuit of basic science even as "fundamental and important questions regarding health services, psychosocial treatments, conceptual issues, public health, and patient initiatives remain marginally funded" (Sadler 2013, 29), it remains to be seen whether the NIMH will be truly ecumenical in the distribution of research dollars across the columns of their matrix.

The RDoC project's purported reductionism differs in an important way from the epistemic bottleneck of the $D S M$, however, insofar as it increases the distance between the laboratory and the clinic rather than collapsing it. If the pretense that psychiatry's scientific and practical objects are one and the same is abandoned, the fits and starts of the NIMH's 
this is a preprint - please ask before citing

descriptive project need not immediately impact clinical nosology, and its reductive approach will not be directly imported into practice. Solomon has argued that while expert disagreement can be generative in science the value of consensus is higher in medicine, where the loss of epistemological authority can be dangerous (Solomon 2014). Her claims are vindicated by the commonly expressed view even the minor modifications of diagnostic categories with each new edition of the DSM can be harmful to patients (Frances 2009). As Schaffner has suggested, clinical research might continue to make progress on refining our methods of patient care while scientists work to reveal the complex and diverse "manymany relations" that make validity such a challenge (Schaffner 2012, 184). However, if the DSM stops playing its role as an epistemic hub (Kutschenko 2011b), the integration of psychiatric knowledge into therapeutics will need to be re-imagined.

\section{Conclusion}

Diverse metaphysical orientations about the nature of diagnostic kinds' kindhood are compatible with the assumption of diagnostic discrimination. Debates among philosophers of psychiatry over psychiatric kinds have focused on appraising these possible metaphysical stances, and there has recently been much effort to resolve the metaphysical nature of psychiatric kinds (Kincaid and Sullivan 2014). Insofar as the objects of diagnostic tests can be seen as either theoretical constructs or real entities, both realists and instrumentalists can beg the question of whether the DSM's diagnostic criteria are indeed discriminative. I conclude by noting that this project has distracted philosophers from the fact that optimism about the discrimination of the diagnostic criteria may, in some or all cases, be unwarranted. If diagnostic discrimination varies across the DSM's 
categories, it renders the question of whether psychiatric kinds are (taken as a class) natural (or human, practical, constructed, etc.) an ill-formed one. Since psychiatrists are, more and more, pursuing piecemeal causal explanations about constructs below the level of diagnoses, philosophers should follow Kincaid (2008) in leaving the question of diagnostic kindhood behind. Instead, philosophers can investigate the ways in which psychiatry stabilizes its diverse objects of research across disciplinary boundaries in the absence of the DSM's authoritative voice (Sullivan 2014).

\section{Acknowledgements}

Grateful thanks for feedback on this material are due to the participants of the IASPM (Paris 2013), the members of STARS, Lara Keuck, Peter Machamer, Ken Schaffner, Serife Tekin, Jon Tsou, Eric Turkheimer, Adam Weg, and especially Maël Lemoine, Joe McCaffrey, Jeff Poland, and an anonymous reviewer. 
this is a preprint - please ask before citing

\section{References}

Andreasen, Nancy. 1995. "The Validity of Psychiatric Diagnosis: New Models and Approaches." America Journal of Psychiatry 152:161-62.

-----. 2007. "DSM and the Death of Phenomenology in America: An Example of Unintended Consequences." Schizophrenia Bulletin 33 (1):108-12.

Bolton, Derek. 2013. "Should Mental Disorders Be Regarded as Brain Disorders? 21st Century Mental Health Sciences and Implications for Research and Training." World Psychiatry 12 (1): 24-25.

Bowker, Geoffrey C., and Susan Leigh Star. 1999. Sorting Things Out: Classification and Its Consequences. Cambridge: MIT Press.

Feighner, John, Eli Robins, Samuel Guze, Robert A. Woodruff, George Winokur, and Rodrigo Munoz. 1972. "Diagnostic Criteria for Use in Psychiatric Research.” Archives of General Psychiatry 26:57-63.

First, Michael B, and Drew Westen. 2007. "Classification for Clinical Practice: How to Make ICD and DSM Better Able to Serve Clinicians." International Review of Psychiatry 19 (5):473-81.

Frances, Allen J. 2009. "A Warning Sign on the Road to DSM-V: Beware of Its Unintended Consequences." Psychiatric Times 26 (8):1-4.

-----. 2013. “The Role of Biological Tests in Psychiatric Diagnosis.” Huffington Post: Science. July 25.

Gillihan, Seth J, and Erik Parens. 2011. “Should We Expect 'Neural Signatures' for DSM Diagnoses?" The Journal of Clinical Psychiatry 72 (10): 1383-89.

Hyman, Steven E. 2010. "The Diagnosis of Mental Disorders: The Problem of Reification.” Annual Review of Clinical Psychology 6:155-79.

Hyman, Steven E., and Wayne S. Fenton. 2003. "What Are the Right Targets for Psychopharmacology?" Science January 17: 350-51.

Insel, Thomas R. 2013. “Transforming Diagnosis.” Director's Blog, NIMH.

Kendler, Kenneth S. 1980. "The Nosologic Validity of Paranoia (Simple Delusional Disorder): A Review.” Archives of General Psychiatry 37:699-706.

Kendler, Kenneth S., and R.A. Muñoz. 2010. "The Development of the Feighner Criteria: A Historical Perspective." The American Journal of Psychiatry 167:134-42. 
Kendler, Kenneth S., Peter Zachar, and Carl Craver. 2009. "What Kinds of Things Are Psychiatric Disorders?” Psychological Medicine 41:1143-50.

Kincaid, Harold. 2008. "Do We Need Theory to Study Disease?" Perspectives in Biology and Medicine 51: 367-78.

Kincaid, Harold, and Jacqueline A Sullivan. 2014. Classifying Psychopathology. Cambridge, MA: MIT Press

Knottnerus, J. A., and Buntinx, Frank. 2009. The Evidence Base of Clinical Diagnosis: Theory and Methods of Diagnostic Research. West Sussex: Blackwell.

Kupfer, David J., Michael B. First, and Darrel A. Regier. 2008. A Research Agenda for DSM-V. Arlington: American Psychiatric Publishing.

Kutschenko, Lara K. 2011a. “In Quest of 'Good' Medical Classification Systems.” Medicine Studies.

Kutschenko, Lara K. 2011b. "How to Make Sense of Broadly Applied Medical Classification Systems: Introducing Epistemic Hubs." History and Philosophy of the Life Sciences 33 (4):583-601.

McLaren, Niall. 2011. "Cells, Circuits, and Syndromes: a Critical Commentary on the NIMH Research Domain Criteria Project." Ethical Human Psychology and Psychiatry 13 (3):229-36.

Meehl, Paul E. 1986. “Diagnostic Taxa as Open Concepts: Metatheoretical and Statistical Questions About Reliability and Construct Validity in the Grand Strategy of Nosological Revision." In Millon, Theodore, and Gerald L Klerman, eds. Contemporary Directions in Psychopathology, 215-31. New York: Guilford.

Merikangas, Kathleen Ries, and Neil Risch. 2003. "Will the Genomics Revolution Revolutionize Psychiatry?" American Journal of Psychiatry 160 (4): 625-35.

Nandi, Arijit, John R Beard, and Sandro Galea. 2009. "Epidemiologic Heterogeneity of Common Mood and Anxiety Disorders Over the Lifecourse in the General Population: A Systematic Review." BMC Psychiatry 9:31.

Olbert, Charles, Gala, Gary J., and Larry A. Tupler. 2014. “Quantifying Heterogeneity Attributable to Polythetic Diagnostic Criteria: Theoretical Framework and Empirical Application." Journal of Abnormal Psychology.

Parnas, Josef, Paul Møller, Tilo Kircher, Jørgen Thalbitzer, Lennart Jansson, Peter Handest, and Dan Zahavi. 2005. "EASE: Examination of Anomalous Self-Experience."

Psychopathology 38 (5):236-58. 
Poland, Jeffrey, and Barbara Von Eckardt. 2013. "Mapping the Domain of Mental Illness." In K.W.M Fulford, Martin Davies, Richard Gipps, George Graham, John Sadler, Giovanni Stanghellini, and Tim Thornton, eds., Oxford Handbook of Philosophy and Psychiatry, 735-52. Oxford: Oxford University Press.

Robins, E., and Samuel Guze. 1970. "Establishment of Diagnostic Validity in Psychiatric Illness: Its Application to Schizophrenia." American Journal of Psychiatry 126 (7):98387.

Ross, Don. 2014. "Syndrome Stabilization in Psychiatry: Pathological Gambling as a Case Study." In Classifying Psychopathology, Jacqueline A Sullivan and Harold Kincaid, eds., 195-208. Cambridge, MA: The MIT Press.

Sadler, John Z. 2013. "Considering the Economy of DSM Alternatives." In Making the DSM-5: Concepts and Controversies, ed. Joel Paris and James Phillips, 21-38. New York: Springer.

Sanislow, Charles A., Daniel S. Pine, Kevin J. Quinn, Michael J. Kozak, Marjorie A. Garvey, Robert K. Heinssen, Philip Sung-En Wang, and Bruce N. Cuthbert. 2010. "Developing Constructs for Psychopathology Research: Research Domain Criteria." Journal of Abnormal Psychology 119 (4):631-39.

Sass, Louis A., Josef Parnas, and Dan Zahavi. 2011. "Phenomenological Psychopathology and Schizophrenia: Contemporary Approaches and Misunderstandings." Philosophy Psychiatry and Psychology 18 (1):1-23.

Schaffner, Kenneth F. 2012. A Philosophical Overview of the Problems of Validity for Psychiatric Disorders. In Philosophical Issues in Psychiatry II: Nosology, eds. Kenneth S. Kendler and Josef Parnas, 169-89. Oxford: Oxford University Press.

Solomon, Miriam. 2014. "Expert Disagreement and Medical Authority." In Philosophical Issues in Psychiatry III: The Nature and Sources of Historical Change, eds. Kenneth S. Kendler and Josef Parnas. Oxford: Oxford University Press.

Sullivan, Jacqueline. 2014. "Stabilizing Mental Disorders: Prospects and Problems." In Classifying Psychopathology, Jacqueline A Sullivan and Harold Kincaid, eds., 195-208. Cambridge MA: The MIT Press.

Vaillant, George E. 1984. "The Disadvantages of DSM-III Outweigh Its Advantages." American Journal of Psychiatry 141 (4). American Psychiatric Association: 542-45.

Walter, Henrik. 2013. "The Third Wave of Biological Psychiatry." Frontiers in Psychology 4: Article 582. 
this is a preprint - please ask before citing

Whooley, Owen. 2010. "Diagnostic Ambivalence: Psychiatric Workarounds and the Diagnostic and Statistical Manual of Mental Disorders." Sociology of Health \& Illness 32 (3):452-69. 